\title{
Miocarditis eosinofílica como causa de disfunción ventricular izquierda reversible
}

\section{María Juliana Rodríguez-González ${ }^{\mathrm{a}, \mathrm{b}, \mathrm{c}, \mathrm{d}, *}$, Malka Irina Wadnipar-Gutiérrez ${ }^{\mathrm{e}}$ y Lauren Sofía Calvo-Betancourt ${ }^{\mathrm{e}}$}

\author{
a Universidad El Bosque, Fundación Clínica Abood Shaio, Bogotá, Colombia \\ b Universidad de Barcelona, Hospital del Mar, IMAS, Barcelona, España \\ c Massachussets General Hospital, Boston, Massachussets, Estados Unidos \\ d Instituto del Corazón de Floridablanca, Fundación Cardiovascular de Colombia, Floridablanca, Santander, Colombia \\ e Fundación Cardiovascular de Colombia, Floridablanca, Colombia
}

Recibido el 26 de agosto de 2015; aceptado el 10 de enero de 2016

Disponible en Internet el 28 de febrero de 2016

\section{PALABRAS CLAVE \\ Miocarditis; \\ Falla cardiaca aguda; \\ Falla cardiaca; \\ Dispositivo de \\ asistencia cardiaca}

\begin{abstract}
Resumen La miocarditis eosinofílica es una entidad poco difundida ya que su diagnóstico histopatológico se realiza en casos de mayor severidad, donde la biopsia endomiocárdica es mandatoria. No obstante, dado que esta entidad suele superponerse a muchas patologías: las infecciosas, las inmunológicas, o asociadas a la hipersensibilidad y esto se asocia con la reversibilidad, es importante tenerla presente como entidad nosológica en cuadros agudos de la disfunción ventricular izquierda.

Puede o no estar asociada a la eosinofilia periférica, su ausencia no descarta la enfermedad. Se presenta un paciente joven (35 años de edad), con un cuadro clínico de la falla cardiaca aguda que progresa rápidamente a choque cardiogénico con poca respuesta al manejo inicial instaurado (inodilatadores, vasopresores), requiriendo el uso de soporte circulatorio extracorpóreo. Como antecedente: la reciente finalización del tratamiento para leishmaniasis mucocutánea, con antimoniato de meglumina (glucantime). Informe del servicio de patología: miocarditis eosinofílica.

Se logra el destete del soporte circulatorio, presenta mejoría de la función sistólica del ventrículo izquierdo con recuperación total del cuadro clínico. Este caso es relevante en cuanto a diagnóstico de disfunción ventricular aguda, con una asociación previamente no descrita con antimoniato de meglumina (glucantime), nos recuerda la importancia de realizar: la biopsia endomiocárdica para definir la etiología, la toma de decisiones de terapias avanzadas, conociendo que en ciertas circunstancias puede presentarse reversibilidad de la disfunción miocárdica y mejoría del cuadro clínico.

(c) 2016 Sociedad Colombiana de Cardiología y Cirugía Cardiovascular. Publicado por Elsevier España, S.L.U. Este es un artículo Open Access bajo la licencia CC BY-NC-ND (http:// creativecommons.org/licenses/by-nc-nd/4.0/).
\end{abstract}

\footnotetext{
* Autor para correspondencia.

Correo electrónico: juli752000@yahoo.com (M.J. Rodríguez-González).
} 


\section{KEYWORDS}

Myocarditis; Acute heart failure; Heart failure; Cardiac assist device

\section{Eosinophilic myocarditis as a cause of reversible left ventricular dysfunction}

Abstract Eosinophilic myocarditis is a little-known entity as its histopathological diagnosis is conducted in the most severe cases, where an endomyocardial biopsy is mandatory. However, because this condition is usually overlaps with many pathologies -infectious, immunological, or associated to hypersensitivity, and this is related to reversibility - it is important to keep it in mind as a nosological entity in acute presentations of left ventricular dysfunction.

It may or may not be associated to peripheral eosinophilia, though its absence does not rule out the condition.

A case of a young male patient ( 35 year-old) with clinical features of acute cardiac failure which progresses rapidly into cardiogenic shock with low response to initial management (inodilators, vasopressors) requiring the use of extracorporeal circulation support is presented. Previous history: recent completion of treatment for mucocutaneous leishmaniases with meglumine antimoniate (glucantime). Pathology service report: eosinophilic myocarditis.

Weaning from circulatory support is achieved, presenting an improvement of systolic function of the left ventricle with complete recovery of the symptomatology. This case is relevant due to the diagnosis of acute ventricular dysfunction with an association with meglumine antimoniate (glucantime) that was previously not described. It reminds of the importance of carrying out an endomyocardial biopsy in order to define the aetiology and the decision-making on advances therapies, knowing that in some circumstances there can be a reversibility of the myocardial function and an improvement of the symptomatology.

(c) 2016 Sociedad Colombiana de Cardiología y Cirugía Cardiovascular. Published by Elsevier España, S.L.U. This is an open access article under the CC BY-NC-ND license (http:// creativecommons.org/licenses/by-nc-nd/4.0/).

\section{Introducción}

La insuficiencia cardiaca es una de las patologías crónicas con mayor morbimortalidad en nuestro medio, implicando un gran gasto para cualquier sistema de salud ${ }^{1}$. Por esta razón, se han desarrollando programas cuya finalidad es la implementación de estrategias que han logrado impactar en sobrevida, hospitalizaciones y mejoría de parámetros fisiológicos como: la clase funcional, la función ventricular y los marcadores de distensión, inflamación y fibrosis cardiaca.

Sin embargo, en el territorio de la falla cardiaca aguda hay un sin número de patologías para las cuales, además, del bloqueo neurohormonal se deben dirigir terapias específicas de soporte multiorgánico, al igual que algoritmos diagnósticos con el fin de mejorar la sobrevida en este grupo de pacientes.

La miocarditis eosinofílica es una de las etiologías del síndrome de la falla cardiaca aguda (choque cardiogénico) ${ }^{2}$, que cursa con disfunción ventricular reversible, generalmente, asociada a la eosinofilia periférica ${ }^{2}$; por lo que suele superponerse con un sinnúmero de patologías infecciosas o de hipersensibilidad ${ }^{2}$. Por esta razón, es importante conocer las situaciones que cursan con este cuadro de miocarditis eosinofílica mediante el uso de estrategias diagnósticas que nos permitan realizar cambios terapeúticos y lograr mejorar los desenlaces ${ }^{1-7}$.

Este reporte busca concientizar a la población médica sobre la presencia de procesos patológicos agudos y reversibles a nivel miocárdico que nos deben hacer actuar rápidamente para disminuir la mortalidad y morbilidad asociadas.

\section{Caso clínico}

Hombre de 35 años de edad, con cuadro clínico de 4 días de evolución caracterizado por dolor precordial opresivo de intensidad 6/10 en la escala análoga del dolor, progresivo, asociado a diaforesis y dificultad respiratoria, por lo que consulta a hospital local en su ciudad de origen; no existen condiciones cardiovasculares previamente conocidas. Antecedente de importancia dado por noxa epidemiológica de picadura por artrópodos en diciembre de 2014, el cual se manejó como leishmaniasis mucocutánea con antimoniato de meglumina (glucantime) por 20 días (IM 136 mg día), completó esquema 15 días previos al inicio de los síntomas.

Dentro de los estudios realizados: un electrocardiograma con evidencia de isquemia lateral baja, biomarcadores positivos (troponina I: 2,47, CK: 428, CK-MB: 64), TSH: 3,98, BNP: 2500, hemoglobina: 12,5 , hematocrito: 36,5 , leucocitos: 16040 , neutrofilos: $87 \%$, monocitos: 2,8 , linfocitos: 9,3 , plaquetas: 337000 , VIH: negativo, VDRL: no reactiva, ECO TT con FE 15\%. Se inicia manejo antifalla cardiaca, no obstante, el paciente evoluciona a choque cardiogénico refractario al tratamiento vasodilatador e inodilatador por lo cual se activa grupo de la falla cardiaca avanzada y se activa el ECMO móvil y se traslada a nuestra institución.

Ingresa a nuestra institución en muy malas condiciones, en choque cardiogénico refractario por lo cual se consideró candidato a soporte venoarterial con ECMO, con adecuada tolerancia y respuesta. Paraclínicos de ingreso a nuestra institución: Eco TT: ventrículo izquierdo de diámetro normal, deterioro difuso de la contractilidad, paredes gruesas. FE del 20\%, BNP: 1430, HB: 10,8, HTO: 30,2, LEU: 11,3, NEU: $78 \%$, EOS: $1,8 \%$ (tabla 1). Se le realiza biopsia endomiocárdica, la 


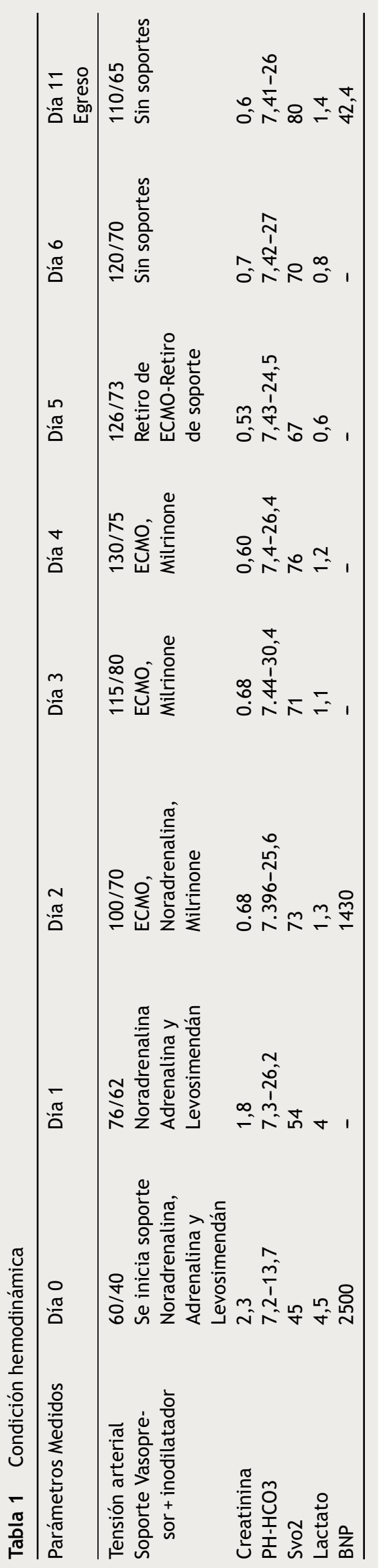

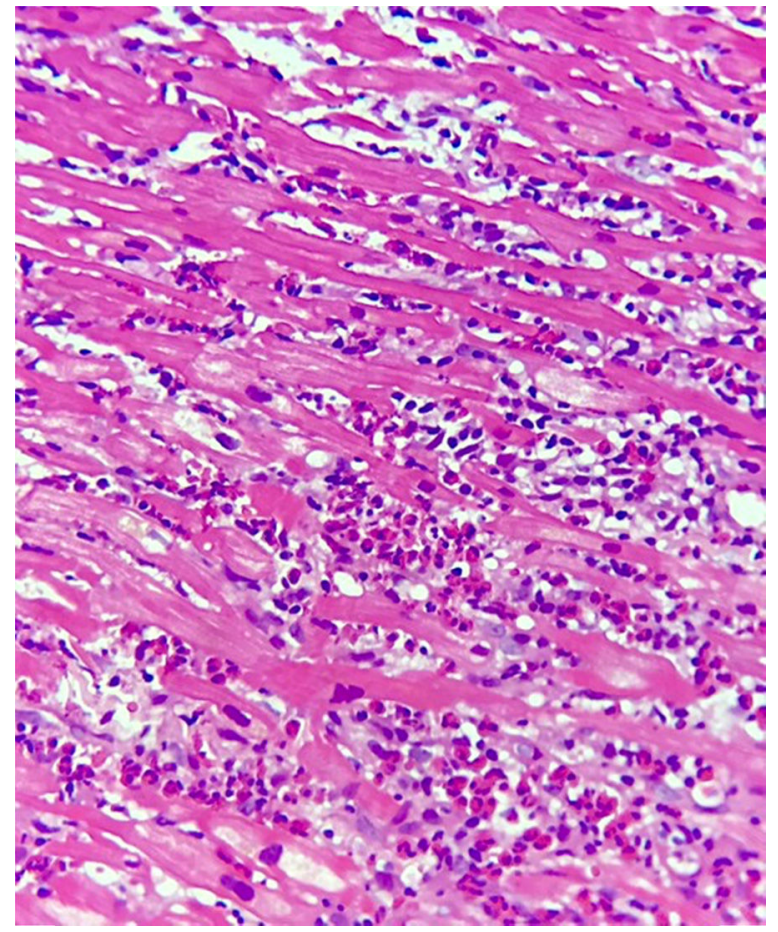

Figura 1 Corte histopatológico con H\&E. Tejido de arquitectura irregular con presencia de severo infiltrado dado por eosinófilos y linfocitos maduros.

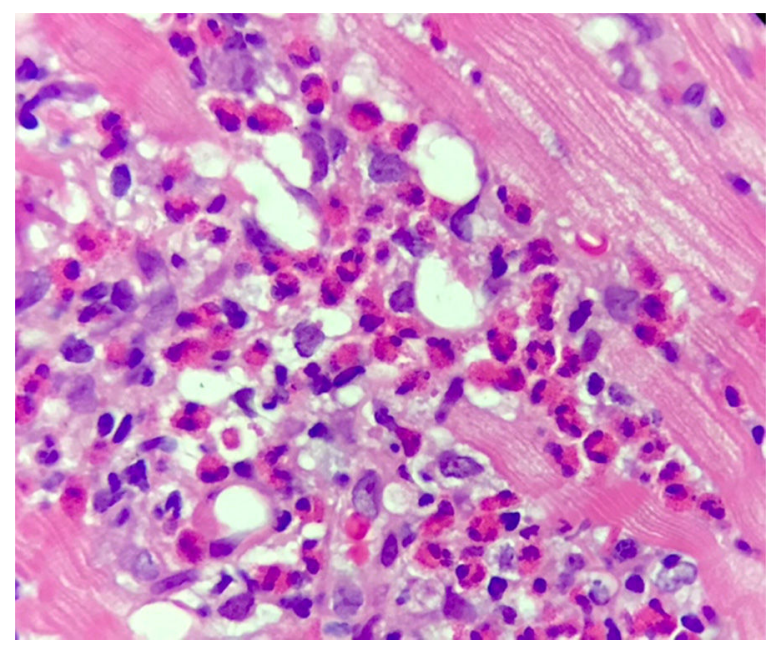

Figura 2 Corte histopatológico. Formación de abscesos eosinofílicos y daño miocitario.

cual reporta miocarditis eosinofílica sin fibrosis según criterios de Dallas (figs. 1 y 2). Reporte de anticuerpos, strout y biopsia negativos para Chagas. Teniendo en cuenta adecuada tolerancia, mejoría de las condiciones hemodinámicas y recuperación de la función sistólica del ventrículo izquierdo se decidió retiro de ECMO al tercer día lográndose destete exitoso. Se realizó ECO TT de control con mejoría de la FE (30\%), respecto al ingreso, BNP de control de 41 . Se realizó retiro de infusiones de soporte con adecuada tolerancia, sin signos de bajo gasto, asintomático cardiovascular, gasto urinario adecuado. Previo al egreso se decide realizar nuevo ECO TT de control al día 10 con evidente recuperación de 
Tabla 2 Controles de la ecocardiografía

\begin{tabular}{llll}
\hline & $\begin{array}{l}\text { Eco TT } \\
\text { (Ingreso) }\end{array}$ & $\begin{array}{l}\text { Eco TT } \\
\text { (Retiro Ecmo) }\end{array}$ & $\begin{array}{l}\text { Eco TT } \\
\text { (Salida) }\end{array}$ \\
\hline FE\% & $20 \%$ & $33 \%$ & $55 \%$ \\
NYHA & IV & II-III & I \\
Hallazgos & Diámetro del vI & Diámetro del vi y VD & Diámetro del vI y VD \\
& normales. VTI $14 \mathrm{~cm} / \mathrm{seg}$ & normal. VTI $15 \mathrm{~cm} / \mathrm{seg}$ & normal. VTI $18 \mathrm{~cm} / \mathrm{seg}$ \\
& Derrame pericárdico de & Derrame pericárdico & Derrame pericárdico \\
& aprox. $150 \mathrm{cc}$. & aprox. $100 \mathrm{cc}$. & aprox. $100 \mathrm{cc}$. \\
\hline
\end{tabular}

la función ventricular FE 55\% (tabla 2). Teniendo en cuenta la adecuada evolución clínica del paciente, con recuperación de la función ventricular y adecuada tolerancia a la medicación se considera dar egreso al paciente.

\section{Discusión}

La miocarditis eosinofílica corresponde al tejido miocárdico inflamado con un gran componente de infiltración por eosinófilos; lo cual le da su nombre histopatológico. Esta entidad puede estar o no asociada a la eosinofilia periférica sin cambiar esto el diagnóstico patológico ${ }^{1-3}$.

Las etiologías de esta descripción patológica son múltiples; pero las más enumeradas en la literatura corresponden a un síndrome de miocarditis por hipersensibilidad o a un síndrome hipereosinofílico ${ }^{2,3}$.

La miocarditis por hipersensibilidad es una rara entidad que se ha descrito asociada a múltiples procesos patológicos que pueden ser: infecciosos, autoinmunes o estar asociados a reacciones alérgicas a medicamentos ${ }^{2,3}$.

La primera descripción de este patrón histopatológico fue documentada por French AJ, y Cols., como una respuesta idiosincrática a las sulfonamidas, sin embargo, se encontró el mismo patrón histopatológico en ratas con aplicación intraperitoneal de esta medicación ${ }^{2,3,5}$. Posteriomente, hubo más descripciones de este patrón asociado a otros compuestos: acetazolamida, amitriptilina, anfotericina B, ampicilina, carbamazepina, cefaclor, cloranfenicol, clortalidona, desipramina, hidroclorotiazida, indometacina, interleuquina-4, isoniazida, metildopa, oxifenilbutazona, ácido para aminosalicílico, penicilina, fenobarbital, fenilbutazona, fenitoína, espironolactona, estreptomicina, sulfadiazina, sulfisoxazol, sufonilureas, toxoide tetánico y tetraciclina ${ }^{2,3,5,6}$.

Por otra parte, está el síndrome hipereosinofílico; la segunda etiología más común asociada a miocarditis eosinofílica. Descrito como una enfermedad linfoproliferativa de etiología incierta no considerada patología clonal o leucemia. Definida por la presencia de la eosinofilia periférica $>1500 /$ ul durante por lo menos 6 meses, no asociada a patología subyacente que produzca la eosinofilia ${ }^{3}$.

Se caracteriza por la infiltración de múltiples órganos como: el cerebro, el intestino, el bazo, el hígado, la médula ósea y el corazón ${ }^{3}$. De ahí la gran variedad de presentaciones clínicas. El pronóstico adverso es mayormente explicado por el compromiso cardiaco y la presencia de la falla cardiaca como desenlace final; principalmente, en el contexto de cronicidad y progresión del cuadro de la falla cardiaca a etapas terminales de la misma o a requerimiento de procedimientos de terapia avanzada ${ }^{3}$.

En el síndrome hipereosinofílico el compromiso cardiaco ocurre hasta en un 80 a $85 \%$ de las veces, y presenta tres estadios $^{2,3}$. En múltiples series se ha observado un peor pronóstico sin manejo, y la peor sobrevida se evidencia en los pacientes que desarrollan la falla cardiaca y el conteo de blancos por encima de 90.000 con algunas células inmaduras en la sangre ${ }^{2,3}$. En esta última entidad se han realizado algunas descripciones de casos con manejos que van desde el uso de esteroides con mejorías importantes, el uso de la hidroxiurea y finalmente el uso de los inmunosupresores con porcentajes de mejoría hasta del $40 \%{ }^{2,3,8}$.

La sintomatología en la miocarditis eosinofílica depende de la etiología. Puede abarcar desde: cefalea, osteomialgias, rash con posterior presencia de signos y síntomas de la falla cardiaca hasta un cuadro severo del choque cardiogénico; todo esto asociado a la ingesta o suspensión reciente (pocos días o meses) de un posible agente causal ${ }^{7}$. En nuestro reporte llama la atención la ingesta previa de antimoniato de meglumina.

En los paraclínicos podemos encontrar alteraciones en los marcadores de injuria miocárdica, y alteraciones electrocardiográficas que no son específicas de este diagnóstico (alteraciones del segmento ST, alteraciones en la conducción intraventricular, de la conducción AV, arritmias asociadas del tipo arritmias supraventriculares, ventriculares $)^{2,3,5}$.

Su diagnóstico se basa en un alto grado de sospecha en el contexto de un paciente con la falla cardiaca aguda y el deterioro progresivo de sus parámetros hemodinámicos, teniendo en cuenta la severidad del compromiso miocárdico ${ }^{2,3}$.

El gold estándar para el diagnóstico es la biopsia endomiocárdica. No obstante, se ha considerado que la caracterización del tejido mediante la resonancia magnética nuclear podría en algunos casos orientar el diagnóstico etiológico o la toma de la biopsia endomiocárdica ${ }^{7}$. Siendo claro que la toma de la biopsia endomiocárdica en el paciente no críticamente comprometido no es una recomendación $\mathrm{IA}^{7}$.

En nuestro caso llama la atención que en las series de casos revisadas no se había descrito la asociación con esta medicación (antimoniato de meglumina). No obstante, esto puede deberse a que la leishmaniasis en endémica en nuestras regiones de Centroamérica y Suramérica comparándola con América del Norte; de donde tenemos los principales reportes.

En cuanto al tratamiento instaurado hay una gran variedad de opciones terapéuticas basadas en descripciones de casos aislados, por lo cual no existe un consenso; se han 
utilizado esteroides con buenos resultados o simplemente la suspensión del agente cruento sospechado y el soporte hemodinámico teniendo en cuenta lo avanzado del compromiso miocárdico ${ }^{2-5}$.

\section{Conclusión}

Según nuestra revisión, este sería el primer caso reportado de miocarditis eosinofílica asociada al uso de antimoniato de meglumina (glucantime) como causa de disfunción ventricular izquierda reversible. En nuestro medio es importante considerar este diagnóstico en el contexto de un paciente con falla cardiaca aguda una vez descartadas las causas más comunes ya que su potencial reversibilidad tiene implicaciones terapéuticas y pronósticas.

\section{Responsabilidades éticas}

Protección de personas y animales. Los autores declaran que para esta investigación no se han realizado experimentos en seres humanos ni en animales.

Confidencialidad de los datos. Los autores declaran que en este artículo no aparecen datos de pacientes.

Derecho a la privacidad y consentimiento informado. Los autores declaran que en este artículo no aparecen datos de pacientes.

\section{Conflicto de intereses}

Los autores declaran no tener ningún conflicto de intereses.

\section{Agradecimientos}

Servicio Patología Fundación Cardiovascular de Colombia.

\section{Bibliografía}

1. Fonarow Gregg C, Adams Kirkwood F Jr, Abraham William T, Clyde W, Yancy W, John Boscardin, PhD for the ADHERE Scientific Advisory Committee, Study Group, and Investigators. Risk Stratification for In-Hospital Mortality in Acutely Decompensated Heart Failure. Classification and Regression Tree Analysis. JAMA. 2005;293:572-80.

2. Fredric Ginsberg, MDa,T, Joseph E. Parrillo, MD. Eosinophilic Myocarditis, Heart Failure Clin. 2005; 1:419-429.

3. Houman Rezaizadeh, Mónica Sánchez-Ross, Edo Kaluski, Marc Klapholz, Bunyad Haider, Christine Gerula. Acute eosinophilic myocarditis: Diagnosis and treatment. Acute Cardiac Care. 2010;12:31-6.

4. Il Suk Sohn, M.D., Jong Chun Park, M.D, Jae Hun Chung, M.D, Kye Hun Kim, M.D, Youngkeun Ahn, M.D, Myung Ho Jeong, M.D. and Jeong Gwan Cho, M.D, A Case of Acute Eosinophilic Myopericarditis Presenting with Cardiogenic Shock and Normal Peripheral Eosinophil Count. The Korean Journal of Internal Medicine. 2006; 21:136-140.

5. Thierry Fozing, Nayef Zouri, Axel Tost, Rainer Breit, Gottfried Seeck, Charlotte Koch, Cem Oezbek. Management of a Patient With Eosinophilic Myocarditis and Normal Peripheral Eosinophil Count: Case Report and Literature Review. Circ Heart Fail. 2014;7:692-4.

6. French AJ, Weller CV. Interstitial myocarditis following the clinical and experimental use of sulfonamide drugs. Am J Pathol. 1942;18:109-21.

7. Leslie T. Cooper, MD, FAHA, FACC; Kenneth L. Baughman, MD, FAHA, FACC; Arthur M. Feldman, MD, PhD, FAHA, FACC; Andrea Frustaci, MD; Mariell Jessup, MD, FAHA, FACC; Uwe Kuhl, MD; Glenn N. Levine, MD, FAHA, FACC; Jagat Narula, MD, PhD, FAHA; Randall C. Starling, MD, MPH; Jeffrey Towbin, MD, FAHA, FACC; Renu Virmani, MD, FACC. The Role of Endomyocardial Biopsy in the Management of Cardiovascular Disease. JACC. 2007, Nov. Vol. 50, No. 19, 6:1914-31.

8. Parrillo JE, Fauci AS, Wolff SM. Therapy of the hypereosinophilic syndrome. Ann Intern Med. 1978;89(2):167-72. 